\section{La garantía de sangre segura en las Américas}

\author{
George A. O. Alleyne
}

1 El Dr. Alleyne escribió este mensaje poco tiempo antes de cumplirse su mandato como Director de la Oficina Sanitaria Panamericana el 31 de enero de 2003.
Me es muy grato escribir un mensaje de introducción para este número especial de la Revista Panamericana de Salud Pública/Pan American Journal of Public Health, que está dedicado a los bancos de sangre. Este número no sólo versa sobre los bancos de sangre como sitios encargados de recolectar y procesar sangre, sino que abarca todo el amplio espectro de la medicina transfusional o hemoterapia. La sangre ocupa un lugar especial en la cultura de nuestras civilizaciones como símbolo de fuerza y fuente de vitalidad. Su uso de maneras que hoy en día nos parecen macabras llegó a formar parte de muchos ritos ancestrales. Para todo médico la sangre es objeto de cierta reverencia puesto que hasta ahora no hay nada que la sustituya, y su transfusión puede considerarse el primer transplante de un órgano vital. La historia de la transfusión sanguínea y parte del misterio que la rodea han sido descritos en vivo detalle en uno de los números de la revista Perspectivas, noticiero publicado por la Organización Panamericana de la Salud (OPS). Me hizo gracia una de las anécdotas históricas sobre la transfusión sanguínea. Solemos recordar el año 1942 como aquel en que se produjo el encuentro entre ambos mundos, pero no tanto por haber sido el año en que se practicó la primera transfusión de sangre. Se dice que ese año el Papa Inocencio VIII, víctima de una afección neurológica, recibió una transfusión pero el tratamiento resultó ineficaz y el augusto prelado murió. Afortunadamente, en la historia moderna el tema de la transfusión de sangre ha sido tratado con más benevolencia.

La transfusión sanguínea y los bancos de sangre han sido durante muchos años una de las áreas de cooperación técnica de la OPS. El principal enfoque ha sido el tamizaje de la sangre para prevenir la transmisión de enfermedades, tarea que se consideraba eminentemente como parte del área técnica correspondiente a los servicios de laboratorio. La sección dedicada a los bancos de sangre en la edición de 1998 de la publicación cuadrienal de la OPS titulada $L a$ salud en las Américas se centra casi exclusivamente en el número de unidades de sangre que fueron recolectadas y en las pruebas a las que fueron sometidas para evitar la transmisión de enfermedades. 
Cuando fui reelegido al cargo de Director de la OSP en 1998, insté a la Organización a que se esforzara por lograr que toda la sangre en la Región de las Américas fuese segura, y entre las Orientaciones Estratégicas y Programáticas de la OPS para el cuadrienio de 1999-2002 figuraba el aporte de cooperación técnica "dirigida a mejorar la seguridad y calidad de los procedimientos en los bancos de sangre". Cuando los Cuerpos Directivos de la OPS examinaron el tema en 1999, aprobaron una resolución donde se pedía encarecidamente a los Estados Miembros que "dieran mayor prioridad dentro de sus políticas sanitarias nacionales a la seguridad de la sangre destinada a transfusión". La resolución también resaltaba el desarrollo de programas nacionales de hemoterapia y de servicios de transfusión basados en la donación voluntaria de sangre sin remuneración.
"Me gustaría poder afirmar que hemos
alcanzado la meta de lograr que toda
la sangre en la Región sea segura,
pero no es así. No obstante, ha habido
adelantos muy notables en esa dirección."

En el año 2000, dos acontecimientos fortuitos impulsaron nuestra labor en este campo. El tema del Día Mundial de la Salud 2000 fue "La seguridad de la sangre depende de mí: la sangre segura salva vidas", y ello generó gran entusiasmo en torno a la provisión de sangre segura. Un generoso subsidio de la Fundación de Bill y Melinda Gates le permitió a la OPS redoblar sus esfuerzos en este campo, y ese mismo año la OPS lanzó su Iniciativa Regional sobre Sangre Segura. Me gustaría poder afirmar que hemos alcanzado la meta de lograr que toda la sangre en la Región sea segura, pero no es así. No obstante, ha habido adelantos muy notables en esa dirección.

A mi manera de ver, uno de los adelantos más importantes guarda relación con la manera de concebir la función de la medicina transfusional en el marco de la salud pública. Por años tuve la impresión de que el tema de la sangre gravitaba en torno a su uso clínico y que la medicina transfusional consistía principalmente en saber usar la sangre y sus derivados de manera adecuada. Es posible que la mayor conciencia despertada por la posibilidad de transmitir enfermedades, tales como la infección por VIH y el sida, la enfermedad de Chagas y los diversos tipos de hepatitis, haya llevado el tema de la recolección, tamizaje y uso adecuados de la sangre a cobrar importancia en el terreno de la salud pública. Esto bien puede venir a demostrar que la salud del público, vista individual o colectivamente, no es territorio exclusivo de ninguna disciplina o especialidad en particular.

Me complacen los avances que se están logrando. Hay un sano empeño en conseguir que toda la sangre provenga de donantes voluntarios que no reciben remuneración, como en Aruba, Canadá, Cuba, Curaçao y Estados Unidos de América. La promoción de esta práctica se ve respaldada por pruebas de que hay una mayor incidencia de infecciones cuando la sangre no se obtiene de esta forma. El programa destinado a incrementar las donaciones voluntarias sin remuneración recibe la intensa promoción de grupos locales y de algunos de nuestros socios internacionales, tales como la Cruz Roja. Se basa en datos sociológicos que identifican los valores culturales o creencias que podrían fomentar o frustrar la donación voluntaria. La educación pública orientada a aumentar esta forma de abastecer a un país de la sangre que necesita tiene que estar en sintonía con la cultura particular donde se imparte, puesto que en toda sociedad la sangre es objeto de creencias tenaces. La mayor parte de la sangre obtenida en América Latina y el Caribe sigue proviniendo de donaciones de reemplazo. A pesar de que se trata por todos los medios de desalentar esta práctica, es fácil entender cómo se originó. Pocas sensaciones deben ser tan satisfactorias como la de donar sangre propia no para que acabe almacenada en un vasto depósito amorfo, sino para ayudar a una persona conocida y amada.
"Es imprescindible que la importancia de contar con sangre segura eche raices en la conciencia popular. Como lo expresara el elocuente lema del Día Mundial de la Salud 2000, 'la seguridad de la sangre depende de mí."

Desde luego, debemos seguir promoviendo el tamizaje de la sangre para prevenir la transmisión de enfermedades, y el Programa Regional de Evaluación Externa del Desempeño de la Serología de Infecciones Transmitidas por Transfusión es un elemento crítico para fortalecer la capacidad de los laboratorios nacionales. 
Hemos visto una mayor promoción de pautas y normas para bancos de sangre en los países, así como esfuerzos sostenidos por racionalizar el gran número de bancos de sangre que funcionan actualmente en ellos bajo una gran variedad de esquemas diferentes. El apoyo de la Asociación Estadounidense de Bancos de Sangre ha sido crucial a la hora de establecer programas de estandarización. Debido a que el tamizaje de la sangre ahora se practica con mayor frecuencia, hay menos infecciones transmitidas por transfusión, fenómeno muy claramente documentado en el caso de la enfermedad de Chagas. Me agradan el énfasis en la educación, incluso a distancia, y la diseminación de información técnica y para el público. Es imprescindible que la importancia de contar con sangre segura eche raíces en la conciencia popular. Como lo expresara el elocuente lema del Día Mundial de la Salud 2000, "la seguridad de la sangre depende de mí".

\section{"Siempre podremos aspirar a inventar una sustancia que sustituya a la sangre, pero en el futuro previsible será necesario garantizar que esta fuente de fuerza vital sea lo más segura posible."}

La racionalización, la concesión de licencias y el desarrollo de leyes en torno a los bancos de sangre y su monitoreo deben figurar entre las funciones directivas que competen al Estado por mediación de los ministerios de salud. Al Estado le será difícil manejar adecuadamente todos los bancos de sangre que se necesitan, pero tampoco debe hacerlo, en realidad. El éxito que ha tenido la participación de las organizaciones no gubernamentales en esta área ha sido espectacular, como lo demuestra el caso de la Cruz Roja en Estados Unidos. No obstante, el monitoreo de las condiciones en que se obtiene la sangre, el uso de protocolos y equipos estandarizados y la capacidad para someter la sangre a pruebas de tamizaje deberán seguir siendo, por fuerza, tareas del Estado o tareas expresamente delegadas por este a terceros.

Me ha gustado mucho el enfoque interprogramático de la OPS en relación con la provisión de sangre segura. El éxito en este campo depende mucho de la colaboración entre grupos y programas enfocados en las enfermedades infecciosas, la calidad de la atención y la práctica de laboratorio y, como es lógico, la provisión de sangre segura en situaciones catastróficas es uno de los elementos imprescindibles de la adecuada preparación para enfrentarlas.

En mi opinión, la OPS está tomando medidas apropiadas para ayudar a los países a disponer de sangre segura. Huelga decir que en esto no debe permitirse lasitud alguna. No cabe duda de que la humanidad siempre se verá amenazada por nuevas infecciones y que siempre se enfrentará al reto de determinar si estas infecciones pueden ser transmitidas o no por medio de una transfusión sanguínea. Siempre podremos aspirar a inventar una sustancia que sustituya a la sangre, pero en el futuro previsible será necesario garantizar que esta fuente de fuerza vital sea lo más segura posible.

Puede que llegue el día en que nuestros poetas hablen de la sangre de un modo distinto. Cuando Shakespeare pone en labios de Lady Macbeth las palabras "Make thick my blood", el personaje está deseando cobrar la impunidad y el valor necesarios para llevar a cabo su crimen atroz. En un futuro, es posible que la misma expresión se utilice en conexión con la donación de sangre segura para conseguir que otra persona pueda sobrevivir y disfrutar de la vida. En el entretiempo, sin embargo, todos debemos procurar leer detenidamente el contenido de este número especial de la Revista/Journal y ver cuáles de los magníficos ejemplos que ofrece de programas que han dado resultado se pueden aplicar en cualquier parte de nuestra Región. Se lo recomiendo. 